9th A. Friedmann International Seminar and

3rd Casimir Symposium 2015

International Journal of Modern Physics: Conference Series

Vol. 41 (2016) 1660118 (5 pages)

(C) The Author(s)

DOI: $10.1142 /$ S2010194516601186

\title{
Tetrad currents in the Einstein-Cartan theory
}

\author{
Rostislav F. Polishchuk \\ Lebedev Physics Institute, Russian Academy of Sciences, \\ 53 Leninsky Av., Moscow, 119991, Russia \\ rpol@asc.rssi.ru
}

Received 27 September 2015

Published 18 March 2016

\begin{abstract}
Local and non-local integral conservation laws in the Riemann-Cartan spaces are given. Hypothesis of the friedmons as dark matter particles is proposed. The friedmon mass is about $10^{9} \mathrm{GeV}$, and the symmetry group is dual for $S U(2)$ one.

Keywords: Non-local integral conservation laws; tetrad currents; spin-mass; hypothesis of friedmons; dark matter particles.
\end{abstract}

PACS numbers: $04.20 . \mathrm{CV}$

\section{Tetrad Currents and Conservation Laws}

The gravitational field, as any gauge field, is a connection. The metric of the expanding Universe approaches the de Sitter one. The Casimir operators in this case contain a combination of the spin and mass or spin-mass as total parameter with physical significance due to mixing the spin-mass components under the action of de Sitter group $S O(1,4)$ transformations. ${ }^{1}$ For example, in the supergravity theory with two complex Grassmanian quantities it is eight real coordinates that have a physical significance (or four complex ones — with imaginary time and two space null coordinates) due to its mixing with the expanded Poincaré group transformations. The Einstein equations connect masses with Riemannian curvature of space-time (events' world for Minkowski space). In the framework of Einstein-Cartan theory the spin-mass must be related to the Riemann-Cartan space with the curvature and the torsion of event manifold. ${ }^{2-4}$ There are 16 equations with the non-symmetric Einstein tensor $G_{\mu \nu}$ and the spin tensor $S_{\mu \nu \lambda}$ as a result of the energy-momentum tensor variation with respect to the spinor connection 1-form. The Einstein equivalence principle (gravity and inertia are locally equivalent) is violated: the Cartan

This is an Open Access article published by World Scientific Publishing Company. It is distributed under the terms of the Creative Commons Attribution 4.0 (CC-BY) License. Further distribution of this work is permitted, provided the original work is properly cited. 
torsion is nontrivial for any observers. The Bianchi contracted identities give differential conservation laws for the energy-momentum tensor. There is their non-local integral equivalent in General Relativity ${ }^{5}$ (for example, an integral over space also contains an integral over time). In Riemann-Cartan space-time we deduce the formula $\left(e_{a \mu} d x^{\mu}\right.$ - eigen 1-forms for $T_{(\mu \nu)}$ with the eigenvalues $p_{a}, K_{a \mu \nu}$ are focusing tensors for 3 -forms $* e_{a}, *$ is the Hodge operator) as follows:

$$
\begin{gathered}
G_{\mu \nu} \equiv R_{\mu \nu}-\frac{1}{2} R g_{\mu \nu}=8 \pi G T_{\mu \nu} ; \\
\nabla_{\mu} G^{\mu \nu}=0 ; \\
G_{[\mu \nu]}=4 \pi G\left(\nabla^{\rho} S_{\mu \nu \rho}+S_{\lambda \rho}{ }^{\rho} S_{\mu \nu}{ }^{\lambda}\right) ; \\
T_{(a \mu)}=e_{a}^{\nu} T_{(\nu \mu)}=p_{a} e_{a \mu} ; \\
\int_{\Sigma_{a}\left(s_{a}\right)}\left[* e _ { a } p _ { a } \operatorname { e x p } \int _ { 0 } ^ { s _ { a } } \left(p_{a}^{-1} p^{b} K_{a b b}\right.\right. \\
\left.\left.+\left(4 \pi G p_{a}^{-1}\right) \sqrt{-g_{a a} h_{a}} S_{a}{ }^{\mu \nu}\left(\nabla^{\rho} S_{\mu \nu \rho}+S_{\lambda \rho}{ }^{\rho} S_{\mu \nu}{ }^{\lambda}\right)\right) d s\right]=\text { const. }
\end{gathered}
$$

Here $\Sigma_{a}\left(s_{a}\right)$ are the equidistant cuts $s_{a}=$ const of the world line tubes of matter 3 -volumes $\sqrt{-g_{a a} h_{a}}$, the space-time signature is $(-+++)$, the mass density $p^{0} \geq 0$. But due to the spin repulsing potential an effective density may be negative. The matter collapse will succeed in the limit Planckian matter density and before it in the Cartan radius ${ }^{6} l_{\text {car }}$ (below $J$ is the spin of the particle with Compton length $l_{\text {com }}$, and $l_{p l}$ is the Planckian length):

$$
l_{\text {car }}^{3}=8 \pi J^{2} l_{p l}^{2} l_{c o m}
$$

For example, for the proton $l_{\text {car }} \approx 10^{-26} \mathrm{~cm}$. It is possible also to give the local integral conservation law with the term $\delta d e_{a}$ (a codifferential of the tetrad differential) in the Einstein tensor, contracted with the tetrad $\left(G_{\nu \mu} e_{a}^{\nu}=G_{a \mu}\right)$ giving the tetrad current $^{7} S_{a}$. The corresponding formulae with the Gibbons-Hawking Lagrangian ${ }^{8}$ are:

$$
\begin{gathered}
L=R+2 \delta K=-\nabla_{\mu} e_{a \nu} \cdot \nabla^{\nu} e^{a \mu}+K_{\mu} K^{\mu} \\
K_{\mu}=e_{\mu}^{a} K_{a}, K_{a}=\delta e_{a}=-\nabla^{\mu} e_{a \mu} ; \\
\Delta=\delta d+d \delta, \delta=*^{-1} d * \\
\Delta e_{a}=-\nabla^{2} e_{a}+R_{a}, R_{a}=R_{\mu \nu} e_{a}^{\mu} d x^{\nu} ; \\
G_{\mu \nu}=-\Lambda g_{\mu \nu}+8 \pi G T_{\mu \nu} \Leftrightarrow \\
G_{a}=-\Lambda e_{a}+8 \pi G T_{a} \Leftrightarrow \\
\delta d e_{a}=8 \pi G S_{a}, \delta S_{a}=-\nabla^{\mu} S_{a \mu}=0 ; \\
8 \pi G S_{a}=\left(\Lambda-\nabla^{2}\right) e_{a}+8 \pi G\left(T_{a}-\frac{1}{2} T e_{a}\right)-d K_{a} .
\end{gathered}
$$

Now discuss the gravitational energy problem. The search for the gravitational energy-momentum pseudo-tensor ${ }^{9,10}$ was a mistake: its dependence on the choice of 
coordinates deprives it of a physical significance. It is the reference frame that, as an additional invariant structure on the space-time, that makes sense. It is impossible to introduce the Dirac equations in the Riemann-Cartan spaces and to solve the gravitational energy problem without lowering from the metric level to the tetrad one. Even the accelerating reference frame in flat space-time permits us, due to the Einstein equivalence principle, to speak about a nontrivial gravitation field (without acceleration) with the gravitational energy $-g^{2} / 8 \pi G$ (where $g$ is the free fall acceleration) and with the nontrivial Gibbons-Hawking Lagrangian. Einstein obtained the correct total energy of an island physical system using the asymptotically Cartesian reference frame in the neighborhood of space infinity. ${ }^{11}$ Here the Cartesian coordinates are primary for real translations.

Abraham Leonidovich Zelmanov (1913-1987), my teacher, have been searching unsuccessfully for a chronometric invariant gravitational pseudo-tensor by the method of undetermined coefficients. He introduced the monad vector field and a set of integral time-like world lines as a reference frame. The tetrad field includes the monad field, and the difference $t_{0 \mu}=S_{0 \mu}-T_{0 \mu}$ gives the desired quantity. Of interest is the Steven Weinberg pseudo-tensor ${ }^{12}$ as a difference between the energymomentum tensor and nonlinear parts of the Einstein tensor. But both indices remained coordinate. The tetrad current is an analogical difference, but our contracting the energy-momentum tensor with the tetrad potential is an analogue of the term $A_{\mu} J^{\mu}$ in the framework of electrodynamics. This approach is called semitetrad one: the double contracted Einstein tensor with the tetrad gives 16 scalar equations instead of one tensor equation in Einstein-Cartan theory.

There are many different vacua even in flat space-time related with by the Bogoliubov transformations. Vacuum plus matter is a whole physical system, and the conservation laws must be only realized for vacuum plus matter. ${ }^{13}$ We propose that the gauge constraints for the vacuum background, i.e. $\Gamma$ (connection module "gamma") be a sum of the product of 12 complex Penrose spin coefficients ${ }^{14}$ and complex conjugated ones. The condition $\Gamma=$ min gives a primary reference frame and the corresponding physical vacuum. Vacuum is not invariant under the Lorentz transformations. Due to this non-invariance one speaks about non-localization of the gravitational energy. We introduce the requirement $\delta G_{a}=-\nabla^{\mu} G_{a \mu}=0$ as a conservation gauge for gravitational theory. In this case we have the normal local integral conservation laws in gravity. For the de Sitter world we have:

$$
\begin{gathered}
\delta G_{a}=-\nabla^{\mu} G_{a \mu}=0 ; \\
G_{a}=-\Lambda e_{a}, \\
\delta G_{a}=-\Lambda K_{a}=0 \Leftrightarrow K_{a}=0, L=R=4 \Lambda .
\end{gathered}
$$

Here the conservation gauge gives the Lorentz tetrad gauge (tetrad without dilatation).

The time-form $e_{0}=d t$ implies $S_{00}=0$, but for the Lorentz gauge we obtain the ordinary density for a weak gravitational radiation ${ }^{10}$ and the ordinary negative 
gravitational energy density in a static field. For the Hodge decomposition of tetrad into a sum of gradient, coclosed and harmonic parts

$$
\begin{gathered}
e_{a}=d \alpha_{a}+\delta \beta_{a}+\gamma_{a}, \\
\Delta \gamma_{a}=0,
\end{gathered}
$$

the second term gives a nontrivial gravity and the last term — gravitational radiation.

The Gibbons-Hawking Lagrangian implies the Hamiltonian density as a partial divergence. ${ }^{11}$ Integration in the de Sitter world (a set of 3-spheres with empty cluster set) implies a trivial total mass-energy. It is important that Einstein tensor and energy-momentum one (being proportional to the cosmological term) are nontrivial and being conserved.

The Einstein-Cartan equations admit the interpretation as follows: space-time geometry compensates matter by the Riemannian curvature and the Cartan torsion. The string theory implies string additions (to be transposed to the right side of equations) being important in the origin of Big $\operatorname{Bang}^{15}$ (lower $l$ is the string length, $\alpha^{\prime}$ is the inclination of the Regge trajectory):

$$
\begin{gathered}
G_{\mu \nu}+\Lambda g_{\mu \nu}+\frac{\alpha^{\prime}}{2}\left(R_{\mu \alpha \beta \gamma} R_{\nu}{ }^{\alpha \beta \gamma}-\frac{1}{2} g_{\mu \nu} R_{\alpha \beta \gamma \delta} R^{\alpha \beta \gamma \delta}\right)=8 \pi G T_{\mu \nu} \\
\alpha^{\prime}=l^{2} / 2 \\
G_{\mu \nu}+3\left(\frac{1}{a^{2}}+\frac{\alpha^{\prime}}{a^{4}}\right) g_{\mu \nu}=0 .
\end{gathered}
$$

Here is the string addition for the de Sitter world. If the cosmological term $\left(\Lambda=3 / a^{2}\right)$ is absent then it will arise. The curvature radius must be changed under vacuum transformation when it contributes to the matter mass-energy. The geometric emptiness is forbidden by quantum mechanics, and any real physical vacuum implies a nontrivial geometry. The de Sitter world is a physical system with the nontrivial scalar matter field being conserved.

\section{Conclusion: the Friedmons}

In conclusion, we present the hypothesis of friedmons as dark matter particles. ${ }^{16} \mathrm{It}$ is easy find a connection between the gravitational radius of the Sun $r_{g r}$ and the nucleon size $l_{n}: r_{g r} / l_{n}=l_{n} / l_{p l}, 10^{5} \mathrm{~cm} \cdot 10^{-33} \mathrm{~cm}=\left(10^{-14} \mathrm{~cm}\right)^{2}$. We assume that before the Big Bang the Universe was a 3-sphere (as de Sitter's world) with the limit Planckian density and the gravitational radius $10^{-13} \mathrm{~cm}$ (the initial Lemaitre atom) respectively. Propose that this size is related to the hypothetical particle friedmon $(h / 2 \pi=c=1)$ :

$$
\begin{gathered}
10^{-13} \mathrm{~cm} \cdot 10^{-33} \mathrm{~cm}=\left(10^{-23} \mathrm{~cm}\right)^{2} ; \\
G \cdot\left(2 \cdot 10^{56} \mathrm{~g}\right)=10^{28} \mathrm{~cm} ; \\
m_{f}=0,677 \cdot 10^{9} \mathrm{GeV} .
\end{gathered}
$$


We propose that the symmetry group for the friedmon is the dual $S U(2)$. In Standard model the particles with symmetry groups $S U(3), U(1)$ are stable, but for dual symmetry groups only particles with dual $S U(2)$ group are stable. ${ }^{17,18}$ The interaction between the particles relating to the basic and dual symmetry groups may be only a gravitational one. The friedmons are heavy bi-lepton formations with a quark-type confinement. We propose that the Lemaître atom, after the transformation of topological energetic modes into oscillatory ones, transfers its mass into the ordinary substance. Even if a dust particle contributes its mass $m$ to the de Sitter vacuum, then we shall obtain the de Sitter world with the curvature radius $a$ and the density $\rho: 2 \pi^{2} a^{3} \rho=m$. For the Universe isotropic at large scales its transformation into the scalar field (the de Sitter world) will occur after the decay of all elementary particles. May be there was an approximate equality between total masses of particles and dual particles with the hypothetical Grand Unification group as a product of the $E 8$ and dual one, containing Standard model groups and dual groups as subgroups. The subsequent evolution reduced to predominance of dark matter over the ordinary one. The future quantum gravity theory will give here the final answer.

\section{References}

1. F. Guersey, in Relativity Groups and Topology, ed. C. DeWitt and B. DeWitt (New York, London, 1964).

2. E. Cartan, C. R. Acad. Sci. Paris 174, 593 (1922).

3. T. W. Kibble, J. Math. Phys. 2, 212 (1961).

4. D. W. Sciama, Recent Developments in General Relativity (Pergamon Press, Oxford, 1962).

5. R. F. Polishchuk, Fundamental physical interactions and conservation laws, in Scientifical Edition "Alexandre Gordon", Vol. 1 (2003), p.67, in Russian.

6. R. F. Polishchuk, Post-Einsteinian cosmology: Torsion, strings and dual symmetry, in Seventh International Scientific School "Sciences and Innovations - 2012", July 23-29, 2012 (Yoshkar-Ola, 2012), p. 116, in Russian.

7. R. F. Polishchuk, Grav. Cosmol. 2, 244 (1996).

8. G. V. Gibbons and S. W. Hawking, Phys. Rev. D 15, 2752 (1977).

9. A. Einstein, Sitzungsber. Preuss. Akad. Wiss. 1, 448 (1918).

10. L. D. Landau and E. M. Lifshits, The Theory of Field (Science, Moscow, 1973), p. 360, in Russian.

11. L. D. Faddeev, Uspekhi Fizicheskikh Nauk 136, 435 (1982), in Russian.

12. S. Weinberg, The Quantum Theory of Fields., vol. 2 (Cambridge University Press, Cambridge, 2001).

13. D. A. Kirzhnits and A. D. Linde, Annals of Phys. 101, 195 (1976).

14. R. Penrose and W. Rindler, Spinors ans Space-Time. Vol. 2 (Cambridge University Press, Cambridge, 1986).

15. R. F. Polishchuk, Bulletin of the Lebedev Physics Institute 40, 62 (2013).

16. R. F. Polishchuk, Bulletin of the Lebedev Physics Institute 39, 226 (2012).

17. H.-M. Chan and T. S. Tsou. Acta Physica Polonica B 33, 4000 (2002).

18. R. Penrose, The Road to the Reality. The Complete Guide to the Laws of the Universe (Jonathan Cape, London, 2004). 